\title{
Interstellar Fringes from PSR B0834+06
}

\author{
Barney Rickett ${ }^{1}$, Andrew Lyne ${ }^{2}$ and Yashwant Gupta ${ }^{3}$ \\ 1 University of California, San Diego, CA 92093-0407, USA \\ 2 University of Manchester, Nuffield Radio Astronomy Laboratories, \\ Jodrell Bank, Macclesfield, SK11 9DL \\ ${ }^{3}$ National Centre for Radio Astrophysics, TIFR, Post Bag 3, \\ Ganeshkhind, Pune 411 007, India
}

\section{Observations}

The spectra of radio flux from pulsars show deep modulations, over a typical time scale $\Delta t_{\mathrm{d}}$ and frequency scale $\Delta f_{\mathrm{d}}$. These random diffractive interstellar scintillations are caused by inhomogeneities in the interstellar plasma on scales of $10^{5} \mathrm{~m}$ to $10^{6} \mathrm{~m}$; on occasion there are also refractive modulations caused by very much larger scales. We report here an episode of periodic modulations of the dynamic spectrum of PSR B0834+06 seen in the sequence of observations centered at $408 \mathrm{MHz}$ reported by Gupta et al. (1994 - GRL). There were 12 observations of about 2 hours covering $5 \mathrm{MHz}$ spaced over 14 months. An episode of very fine periodic fringes was detected, though the period $45 \mathrm{kHz}$ in frequency was barely resolved by the $19.5 \mathrm{kHz}$ channel bandwiths; the period in time was about 5 minutes and was fully resolved. The normal $\Delta f_{\mathrm{d}}$ was about 4 $\mathrm{MHz}$ (GRL) - 90 times the fringe period.

The observations are displayed in Figure 2 of Rickett et al 1996 as the 2-d Power spectrum of the original dynamic spectrum. There is a central peak due to diffractive scintillation; it has narrow halfpower widths which are approximately the reciprocals of $\Delta f_{\mathrm{d}}$ and $\Delta t_{\mathrm{d}}$. The power from the fringes is concentrated into the island of power centered at 22 cycles $/ \mathrm{MHz}$ and $0.2 \mathrm{cycles} / \mathrm{min}$. The contours at logarithmic intervals show that the fringe feature is about $20 \mathrm{~dB}$ down from the diffractive peak. This corresponds to a fringe amplitude of about $10 \%$ of the mean flux. The fringe feature was clearly visible on 4 occasions between 9 th Sep 1984 and 9th Oct 1984, and invisible during the following year.

\section{Interpretation and Model for Enhanced Refraction}

Occasional episodes of periodic fringes have been reported by pulsar observers as far back as Ewing et al (1970). Cordes \& Wolszcan (1986 CW) found a simililar episode from PSR B0919+06, also with a large number of fringes across a single "scintle". One can interpret these fringes (Hewish et al 1986), as the interference between two discrete raypaths each of which has been sactter-broadened into an angular radius $\theta_{\mathrm{d}}$. An analysis of how the fringe periods are related to the angles of arrival of the two rays is given by CW. Our 22 cycles/MHz corresponds a difference of $22 \mu \mathrm{secs}$ in the delay beteen the paths. We conclude here that the angle between the raypaths is about 4 mas, at least 20 times $\theta_{\mathrm{d}}$. Goodman et al 
(1987) described how fringes could be caused by a large inner scale. However, in such a scenario any rays or caustics must originate within the envelope of the scattering disc, which is ruled out by the the large ray separation.

From the 30-day duration of the fringe episode we deduce a distance of about $3 \mathrm{AU}$ between the rays; this is very similar to the size deduced in the extreme scattering event reported by Fiedler et al (1987). We follow Romani et al (1987 RBC) in suggesting that the event is caused by enhanced refraction in the line of sight. We suggest that the refracting region does not normally meet the interference condition at the earth, but does on rare occasions, which give rise to multiple rays and fringes. Interference for the $3 \mathrm{AU}$ scale and $4 \mathrm{mas}$ requires a distance $\geq 400 \mathrm{pc}$, consistent with the pulsar's $720 \mathrm{pc}$ distance. When interference occurs there will in general be 3 rays, two of which may easily dominate the flux as in the present case.

Since fringing though unusual is not rare and there is other evidence of enhanced refraction (Rickett, this volume), we suggest regions in a typical line of sight with discrete density inhomogeneities on scales of about $1 \mathrm{AU}$. It seems plausible that these should be identified with the warm ionized medium, as described by Kulkarni \& Heiles (1986) and Reynolds (1988). We postulate filaments of enhanced plasma density controlled by a tangled magnetic field. The AU scale is identified with their typical radius; a density of about $0.25 \mathrm{~cm}^{-3}$ is consistent with pressure balance as proposed by Kulkarni \& Heiles. When a wave passes a layer of this medium it will intersect a number of such filaments, depending on the layer thickness and the filling factor for filaments. We assume that for PSR B0834+06 this number is large compared to unity, making phase modulations with approximately normal statistics. Assuming that 4 mas is, say, 5 times the rms angle of refraction, we require a layer thickness of about $0.6 \mathrm{pc}$ (assuming a filling factor of 0.1). This seems to be compatible with a layer of warm ionized medium surrounding a warm HI cloud. A more detailed description is given by Rickett et al (1996). Very similar geometries have been suggested by RBC and by Wilkinson et al (1994), though not necessarily associated with the warm ionized medium.

\section{References}

Cordes J.M., Wolszczan A., 1986, ApJ, 307, L27, [CW]

Ewing M., Batchelor R., Friefeld R., Price R., Staelin D., 1970, ApJ, 162, L169

Fiedler R.L., Dennison, B., Johnston K. Hewish A., 1987, Nature, 326, 675

Goodman J.J., Romani R., Blandford R., Narayan R., 1987, MNRAS, 229, 73

Gupta Y., Rickett B.J., Lyne A.G., 1994, MNRAS, 269, 1035

Hewish A., Wolszczan A., Graham D.A., 1985, MNRAS, 213, 167

Kulkarni S.R., Heiles C., 1986, "Galactic and Extragalactic Radio Astronomy", eds Kellerman K.I., Verschuur G.L., Springer New York, pp95-152

Reynolds R.J., 1988, ApJ, 333, 341

Rickett B.J., Lyne A.G., Gupta Y., 1996, submitted to MNRAS

Romani R.W., Blandford R.D., Cordes J.M., 1987, Nature, 328, 324 [RBC]

Wilkinson P.N., Narayan R., Spencer R.E., 1994, MNRAS, 269, 67 